\title{
Avances en el tratamiento farmacológico de la hemorragia digestiva de origen oscuro
}

\author{
J. Molina Infante, B. Pérez Gallardo y M. Fernández Bermejo \\ Unidad de Aparato Digestivo. Hospital San Pedro de Alcántara. Cáceres
}

\begin{abstract}
RESUMEN
El desarrollo de la cápsula endoscópica y la enteroscopia de doble balón ha aumentado las tasas de diagnóstico y tratamiento en la hemorragia digestiva de origen oscuro, en la que las angiodisplasias del intestino delgado son la causa más frecuente. Sin embargo, el 25-40\% de los pacientes, no subsidiarios o no respondedores a la terapéutica endoscópica, angiográfica o quirúrgi$\mathrm{ca}$, pueden tener alto riesgo de resangrado y carecen de tratamiento médico claramente eficaz. La utilidad de la terapia hormonal está cuestionada actualmente, sin olvidar los frecuentes y limitantes efectos secundarios. El octreótido suele ser eficaz en el control de la hemorragia pero no parece la terapia de mantenimiento más óptima. Los beta-bloqueantes no selectivos, de igual manera que en la profilaxis de la hemorragia por hipertensión portal, pueden ser útiles en combinación con otros fármacos. Recientemente, el octreotido LAR, una formulación de liberación retardada que se administra una vez al mes por vía intramuscular, y la talidomida oral, un potente inhibidor de la angiogénesis, han demostrado su eficacia y seguridad a largo plazo en casos graves aislados.
\end{abstract}

Palabras clave: Hemorragia digestiva de origen oscuro. Angiodisplasia. Tratamiento hormonal. Octreótido. Octreótido LAR. Beta-bloqueantes. Talidomida. Tratamiento médico.

\begin{abstract}
The development of capsule endoscopy and double-balloon enteroscopy has increased diagnostic and therapeutic rates in obscure gastrointestinal hemorrhage, where angiodysplasia of the small bowel is the most frequent cause. Nevertheless, almost 25$40 \%$ of patients who are not candidates or do not respond to endoscopic, angiographic, or surgical management may be at high risk of rebleeding, and therefore lack a clearly effective medical therapy. The utility of hormonal therapy remains unclear and is burdened by adverse effects. Subcutaneous octreotide usually controls bleeding but does not seem adequate for mainteinance therapy. Non-selective beta-blockers alone or in combination with other treatments, as in the prophylaxis of portal hypertension variceal bleeding, may be helpful. Recently, octreotide LAR, a depot formulation administered once a month intramuscularly, and oral thalidomide, a powerful inhibitor of angiogenesis, have demonstrated their effectiveness and safety for long-term therapy in anecdotal case reports and deserve further investigation.
\end{abstract}

Key words: Obscure gastrointestinal haemorrhage. Angiodysplasia. Hormonal therapy. Octreotide. Octreotide LAR. Thalidomide. Beta-blockers. Medical therapy.

Molina Infante J, Pérez Gallardo B, Fernández Bermejo M. Avances en el tratamiento farmacológico de la hemorragia digestiva de origen oscuro. Rev Esp Enferm Dig 2007; 99: 457-462.

\section{INTRODUCCIÓN}

La hemorragia digestiva de origen oscuro (HDOO) viene definida por la presencia de pérdidas sanguíneas

Recibido: 12-12-06.

Aceptado: 13-12-06.

Correspondencia: Javier Molina Infante. Unidad de Aparato Digestivo. Hospital San Pedro de Alcántara. Avda. Pablo Naranjo, s/n. 10003 Cáceres.e-mail: xavi_molina@hotmail.com del tubo digestivo cuyo origen no puede identificarse con las exploraciones endoscópicas habituales (endoscopia digestiva alta y colonoscopia con intubación ileal). Esta entidad es poco frecuente (5\% de todas las hemorragias digestivas) y se puede manifestar como HDOO oculta, cuando su expresión clínica es la anemia ferropénica o la detección de sangre oculta en heces, o como HDOO manifiesta o patente, en la que se puede constatar de manera recurrente exteriorización hemorrágica en forma de melenas, hematoquecia o rectorragia. La causa más frecuente suelen ser las angiodisplasias del intestino delgado, que 
pueden ser de origen primario o afectar al tubo digestivo en múltiples patologías como la telangiectasia hemorrágica hereditaria, la enfermedad de Von Willebrand, conectivopatías (pseudoxantoma elástico, síndrome de Ehlers-Danlos), la enteritis por radiación, la insuficiencia renal crónica terminal, las cardiopatías valvulares, la hipertensión portal o las vasculitis. La reciente aparición de la cápsula endoscópica y la enteroscopia de doble balón ha permitido el diagnóstico y el tratamiento endoscópico en toda la extensión de una región poco accesible hasta hace unos años; sin embargo, y a pesar de estos prometedores avances, en un $25 \%$ de los casos no se alcanza el diagnóstico combinando ambas exploraciones (1-5). Además de este subgrupo de pacientes sin diagnóstico, un porcentaje variable de los casos diagnosticados pueden no ser candidatos a un manejo agresivo terapéutico por la comorbilidad asociada, por la extensión de las lesiones o por la falta de respuesta de las mismas al tratamiento endoscópico, angiográfico o quirúrgico.

En este contexto, el arsenal farmacológico disponible para la HDOO es limitado, de eficacia cuestionada en algunos casos y la base para su utilización puramente empírica. La baja prevalencia de los pacientes candidatos a tratamiento médico (1-2\% del total de las hemorragias digestivas, si nos atenemos a las cifras mencionadas previamente) hace que los datos existentes en la literatura sean únicamente comunicaciones aisladas de casos clínicos o pequeñas series de pacientes no controladas. Las indicaciones de tratamiento farmacológico de la HDOO, recogidas en el último documento de consenso de la Asociación Americana de Gastroenterología (6), aparecen reflejadas en la tabla I.

Tabla I. Indicaciones de tratamiento médico en la hemorragia digestiva de origen oscuro

1. Pacientes no subsidiarios o no respondedores al tratamiento endoscópico, angiográfico o quirúrgico

2. Lesiones vasculares difusas del intestino delgado o extendidas a tramos superiores o inferiores

3. Lesiones situadas en lugares relativamente inaccesibles para la endoscopia

4. Hemorragia de origen desconocido a pesar de estudio exhaustivo

El propósito de este trabajo, por tanto, es acercar al clínico el mayor número de posibilidades terapéuticas para afrontar el gran reto que casi siempre supone el tratamiento farmacológico de los pacientes con HDOO, muchos de ellos de edad avanzada y comorbilidad asociada e incluso con necesidad de tratamiento antiagregante o anticoagulante que predispone a una mayor riesgo hemorrágico. Algunos de estos fármacos han sido utilizados como terapia combinada en la literatura $(7,8)$.

Los distintos tratamientos que han sido utilizados en la HDOO hasta la fecha están enumerados en la tabla II.
Tabla II. Fármacos utilizados para la hemorragia digestiva de origen oscuro

1. Tratamiento hormonal combinado

2. Análogos de la somatostatina Octreótido Octreótido LAR

3. Beta bloqueantes no selectivos

4. Talidomida

5. Miscelánea (antifibrinolíticos, danazol, desmopresina, fVlla recombinante)

\section{TRATAMIENTO HORMONAL COMBINADO}

El fármaco de elección inicial en el tratamiento de la HDOO ha sido hasta hace pocos años la terapia hormonal. La mejoría observada en la epístaxis de pacientes con telangiectasia hemorrágica hereditaria durante el embarazo y el empeoramiento posterior durante el postparto inmediato fue la observación clínica inicial que aportó el argumento racional para su uso (9). Asímismo, también se objetivó a nivel experimental que el tratamiento hormonal a largo plazo disminuía la hiperemia gástrica en ratas con ligadura mecánica de la vena porta (10). Su efecto no es inmediato, parece ser dependiente de la dosis de estrógenos utilizada y actúa mejorando la circulación, la coagulación y la integridad del endotelio vascular (11). La combinación y las dosis más habituales suele ser etinilestradiol 0,01-0,05 mg y norestisterona 1-3 mg. Esta terapia precisa de periodos de descanso cada 6 meses aproximadamente para minimizar sus notables efectos secundarios, en gran parte dependientes del componente estrogénico (trombosis vasculares, ginecomastia y pérdida de líbido en hombres, metrorragia y tensión mamaria en mujeres). La eficacia del tratamiento hormonal es muy variable según los estudios consultados. Van Cutsem y colaboradores (12), en un ensayo clínico en 9 pacientes con malformaciones vasculares gastrointestinales, demostraron que las necesidades transfusionales eran sustancialmente más bajas cuando los pacientes recibían tratamiento hormonal.

Sin embargo, un estudio posterior con mayor número muestral realizado en pacientes con angiodisplasias intestinales no encontró ninguna ventaja terapéutica en el grupo que recibió tratamiento hormonal combinado (13). La ausencia de beneficio derivado del uso del tratamiento hormonal ha quedado confirmada en el ensayo clínico controlado con placebo de mayor tamaño muestral hasta la actualidad, en el que fueron incluidos 72 pacientes no cirróticos con sangrado crónico por angiodisplasias (14), presentando ambos grupos tasas similares de fallo del tratamiento, resangrado y requerimientos transfusionales. El diseño del estudio fue cuestionado posteriormente debido a la exclusión de pacientes con telangiectasia hemorrágica hereditaria y ectasia vascular asociada a la cirrosis (que hubieran sido los mejores beneficiarios del trata- 
miento hormonal) y por utilizar dosis bajas de etinilestradiol $(15,16)$. A la luz de estos datos, el tratamiento hormonal parece poco eficaz y seguro para el tratamiento de la HDOO, excepto quizá en pacientes con telangiectasia hemorrágica hereditaria, enfermedad de von Willebrand e insuficiencia renal crónica terminal (17) y fuera del espectro de la HDOO en el síndrome de la ectasia vascular antral gástrica $(18,19)$, entidades en las que ha demostrado una eficacia menos cuestionable, controlando el sangrado aunque sin que se apreciaran cambios en el aspecto endoscópico de las lesiones. A pesar de su bajo coste, la reciente aparición de nuevos fármacos eficaces y con mejor perfil de seguridad desplaza a la terapia hormonal de los tratamientos de primera línea para la HDOO.

\section{ANÁLOGOS DE LA SOMATOSTATINA}

El octreótido ha sido históricamente el segundo tratamiento más utilizado tras el fallo del tratamiento hormonal combinado. Es un análogo sintético de la somatostatina que se utiliza debido a su potente efecto vasoconstrictor esplácnico, mediado por la inhibición de péptidos vasodilatadores, que induce una disminución marcada del flujo vascular portal y mesentérico. Asimismo, se ha demostrado experimentalmente en diversos tejidos (ojo, placenta, tumores hepáticos y neuroendocrinos) su capacidad antiangiogénica, aunque se desconoce en la actualidad la relevancia de dicha propiedad para su aplicación práctica en la HDOO.

Ha demostrado su utilidad a la hora de controlar la hemorragia digestiva por angiodisplasias gastrointestinales en pequeñas series no controladas y comunicaciones aisladas, tanto en situaciones agudas (20-22) como en terapia de mantenimiento en sangrados crónicos (23-26), manteniendo la respuesta clínica en algunos casos tras la retirada de la medicación. Ha sido publicado recientemente el primer estudio de cohortes comparado con placebo constatando la eficacia del octreótido a largo plazo en la prevención de la hemorragia por angiodisplasias gastrointestinales (27). La disminución del riesgo de resangrado producida por el octreótido no se vio acompañada de diferencias valorables en los valores del hemograma o requerimientos transfusionales de ambos grupos, aunque sí hubo diferencias significativas en las necesidades de suplementos de hierro.

El octreótido tiene efecto inmediato y se administra por vía intravenosa o subcutánea a unas dosis habituales de $50 \mu \mathrm{g} / \mathrm{h}$ y $50-100 \mu \mathrm{g} / 8-12$ horas respectivamente. La administración por vía subcutánea y una vida media superior a la somatostatina (de 90-100 minutos aproximadamente) han permitido su uso como terapia ambulatoria. Los efectos secundarios vienen derivados fundamentalmente de la inhibición de las secreciones y hormonas del páncreas y tubo digestivo (alteraciones de la glucemia, náuseas, vómitos, estreñimiento, colelitiasis) y son poco frecuentes. La desventaja fundamental de este fármaco a largo plazo es su administración parenteral durante varias veces al día.

El octreótido LAR (long acting release), una formulación de liberación retardada del octreótido, se encuentra indicado actualmente en el tratamiento de la acromegalia y de los tumores neuroendocrinos gastroenteropancreáticos. Presenta las ventajas respecto al octreótido convencional de ser administrado por vía intramuscular una vez al mes, con la misma eficacia y perfil de seguridad y sin precisar ingreso hospitalario, lo que le convierte en una herramienta terapéutica ambulatoria muy atractiva como tratamiento a largo plazo. La eficacia del octreótido LAR en HDOO ha sido únicamente comunicada en tres publicaciones en la literatura, la primera en tres pacientes con sangrado crónico por angiodisplasias difusas de intestino delgado y las otras dos en pacientes con HDOO sin diagnóstico, uno de ellos con enfermedad de von Willebrand $(8,28,29)$. Nuestra Unidad ha publicado recientemente el uso novedoso del octreótido LAR en HDOO grave con altas necesidades trasfusionales en dos pacientes anticoagulados y con comorbilidad severa, uno de ellos sin diagnóstico y el otro con angiodisplasias difusas del intestino delgado (30). Ambos fueron tratados exitosamente durante nueve meses con octreótido LAR sin requerimientos transfusionales, sin efectos secundarios, sin retirada de la anticoagulación y sin interacciones con la misma. La dosis utilizada en todos los casos publicados es 1 vial de $20 \mathrm{mg}$ i.m al mes, sin existir una clara razón para no utilizar las formulaciones de 10 ó $30 \mathrm{mg}$. El principal inconveniente del producto puede ser su coste (desde 700 a $1.100 € \mathrm{al}$ mes, dependiendo de la dosis utilizada) comparado con el tratamiento hormonal y el octreótido convencional. En algunos casos muy específicos como pacientes únicamente candidatos a tratamiento conservador puede ser un tratamiento coste-efectivo, máxime si tenemos en cuenta el ahorro de los gastos de ingresos hospitalarios y pruebas diagnósticas innecesarias, así como el beneficio psicológico para el paciente y su familia derivado del control clínico ambulatorio.

Su excelente perfil de seguridad y comodidad comparado con el resto de tratamientos expuestos es el potencial más atractivo para el uso de octreótido LAR en la HDOO. Queda obviamente por dilucidar la dosis y la pauta de administración del tratamiento a largo plazo con esta molécula, para lo que son necesarios estudios de mayor duración.

\section{BETABLOQUEANTES NO SELECTIVOS}

Esta medicación, de igual modo al que se administra en la profilaxis primaria y secundaria de la hemorragia por varices secundaria a hipertensión portal, trata de controlar el sangrado mediante la disminución del flujo vascular gastrointestinal por vasocontricción esplácnica y reducción de la frecuencia y el gasto cardiaco. Existen casos aislados de tratamiento de gastropatía y colopatía 
hipertensiva grave con betabloqueantes $(19,20)$. Su uso en la literatura para la HDOO es puramente anecdótico, y se ha utilizado a dosis convencionales (60-80 mg/día), en monoterapia o combinado con octreótido LAR, para el tratamiento de angiodisplasias de intestino delgado asociadas a estenosis hipertrófica subaórtica y en HDOO sin diagnóstico $(8,31)$.

\section{TALIDOMIDA}

La talidomida es un fármaco con potente efecto inmunomodulador, antiinflamatorio y antiangiogénico que fue retirado en la década de los años 60 por su teratogenicidad y que está siendo reintroducido en los últimos años para el tratamiento de diversas entidades como la lepra, el mieloma múltiple y diversos tipos de tumores. Se ha identificado el factor de crecimiento del endotelio vascular (FCEV) como el elemento clave para promover la formación del endotelio vascular en las fases iniciales de la angiogénesis. La presencia de altas concentraciones del FCEV provoca una angiogénesis aberrante con formación de angiodisplasias, compuestas por un endotelio vascular con falta de células musculares lisas y, por tanto, susceptible de ruptura. La talidomida ejerce su acción antiangiogénica mediante la inhibición del FCEV.

La talidomida es una opción terapéutica novedosa y prometedora en la HDOO y puede ser utilizada en casos refractarios a la terapia convencional o cuando existe contraindicación a otros tratamientos. Se administra por vía oral a dosis variables de 100 a $300 \mathrm{mg} /$ día sin haberse comunicado efectos adversos reseñables excepto astenia transitoria en los casos comunicados de HDOO, pero la talidomida está contraindicada en pacientes con neuropatía periférica y en mujeres embarazadas o en edad fértil por sus efectos teratógenos y debe utilizarse con cautela en pacientes con enfermedades neurológicas o cardiovasculares y fallo renal o hepático. Además, dada su actividad inmunosupresora mediada por bloqueo del factor de necrosis tumoral, se debe desaconsejar su uso en pacientes con mayor riesgo de infecciones o enfermedades infecciosas crónicas, especialmente en pacientes con VIH. En todos estos casos, el octreótido LAR parece una opción más segura para el tratamiento de la HDOO.

La talidomida ha demostrado recientemente su eficacia en el manejo del sangrado refractario por gastropatía de la hipertensión portal y por proctitis grave post-radioterapia $(32,33)$. Hasta la fecha, existen pocas y muy recientes publicaciones en forma de casos clínicos o series de casos clínicos que evalúan la eficacia de la talidomida en la HDOO. Se ha utilizado con éxito en un paciente con enfermedad de von Willebrand y HDOO por angiodisplasias de intestino delgado, con fracasos terapéuticos previos con ácido tranexámico, desmopresina, octeótrido, factor VII recombinante y tratamiento endoscópico con argón (34), en HDOO por angiodisplasias difusas de intestino delgado (35-37), así como en la anemia crónica por pérdidas digestivas en tres pacientes con enfermedad de Crohn y en tres pacientes con HDOO sin diagnóstico a pesar de estudio (38). Recientemente, Bauditz y colaboradores (39) han estudiado el efecto de la talidomida ( $100 \mathrm{mg} /$ día) durante 3 meses en tres pacientes con sangrado crónico por angiodisplasias de intestino delgado objetivadas por cápsula endoscópica. En todos los casos se consiguió el control de la hemorragia durante un tiempo medio de seguimiento de 2-3 años sin necesidades trasfusionales y se constató una disminución del número de lesiones, del tamaño y de su coloración mediante cápsula endoscópica posterior al tratamiento. Cabe destacar que dado que la talidomida previene la formación ulterior de angiodisplasias, ciclos de tratamiento de varios meses se ven seguidos en todas las comunicaciones de la literatura por 1-2 años al menos de control de la hemorragia.

\section{MISCELÁNEA}

\section{Antifibrinolíticos}

El ácido amino-caproico (AAC) es un potente inhibidor del sistema fibrinolítico que, a dosis bajas, bloquea la conversión de plasminógeno en plasmina. Existe únicamente un caso clínico en el que el AAC, administrado por vía oral a dosis de $1,5 \mathrm{~g}$ dos veces al día, demostró su eficacia en el control del sangrado nasal por malformaciones arteriovenosas en pacientes con telangiectasia hemorrágica hereditaria, aunque no se especificaba si existía sangrado gastrointestinal concomitante (40).

El ácido tranexámico es un fármaco análogo del aminoácido lisina que inhibe la conversión de plasmina a fibrina y que tiene menor potencia antifibrinolítica que el AAC. Ha sido utilizado en pacientes con enfermedad crónica renal con sangrado digestivo por angiodisplasias, a dosis de $10-20 \mathrm{mg} / \mathrm{kg} / 48$ horas, con una duración variable del tratamiento. Queda por determinar si debe ser un tratamiento continuo o intermitente a demanda en los episodios de sangrado agudo $(41,42)$.

El principal riesgo derivado del uso de moléculas antifibrinolíticas son los accidentes trombóticos, por lo que se debe realizar estudio reglado para despistaje de trombofilia previo a su utilización. Los efectos secundarios del AAC y ácido tranexámico son frecuentes y su uso para la HDOO no está apoyado por estudios ensayos clínicos controlados, por lo que los antifibrinolíticos deben ser uno de los últimos escalones terapéuticos para la HDOO y ser indicados, dado su mecanismo de acción, especialmente en paciente con enfermedades hematológicas.

\section{Danazol}

El danazol es un fármaco antigonadotrópico con débil acción androgénica que suprime la producción hipofisaria de FSH y $\mathrm{LH}$, induciendo atrofia del tejido endome- 
trial normal y ectópico. Ha demostrado su eficacia a la hora de controlar hemorragias uterinas funcionales y aquellas asociadas a endometriosis, existiendo dos comunicaciones en la literatura que apuntaban una respuesta parcial a danazol en pacientes con sangrado de origen digestivo y telangiectasia hemorrágica hereditaria sin respuesta a terapia hormonal $(43,44)$, aunque sus secuelas estéticas (acné, hirsutismo, alopecia) e infrecuentes pero graves efectos secundarios (hipertensión intracraneal benigna, trombosis, peliosis hepática, convulsiones) hacen que no sea un fármaco de primera elección en la actualidad.

\section{Desmopresina}

La desmopresina es un análogo sintético de la hormona antidiurética sin efecto vasopresor que ha demostrado aumentar los niveles del factor de von Willebrand (fvW) y factor VIII y mejorar la hemostasia en pacientes con alteraciones funcionales plaquetarias. Está indicado como terapia hemostática en los pacientes con hemofilia A y en la enfermedad de von Willebrand y puede ser administrado por vÍa intravenosa, subcutánea o intranasal. Una comunicación aislada demostró la eficacia de la desmopresina intravenosa para controlar una hemorragia digestiva grave en un paciente con telangiectasia hemorrágica hereditaria y déficit de fvW, permitiendo una colectomía electiva que consiguió la resolución del cuadro (45).

\section{Factor VII recombinante}

El factor VII recombinante activado humano es un activador muy potente de la coagulación que se encuentra actualmente indicado para el tratamiento de la hemorragia en pacientes con hemofilia A y B, con déficit congénito del factor VII y tromboastenia de Glanzmann. Su uso se ha extendido para el control del sangrado a cualquier nivel de la anatomía, con o sin enfermedad hematológica asociada, en situaciones de hemorragia aguda masiva o incontrolable con las medidas habituales. Su corta vida media de 2 horas obliga a una terapia continua con bolos y el control hemostático puede yugular de manera definitiva la hemorragia o ser una medida puente hacia el tratamiento definitivo de la causa. En la hemorragia digestiva, el énfasis ha sido puesto en los pacientes cirróticos con carencias adquiridas de factores de la coagulación, especialmente en el tratamiento de la hemorragia por varices esofágicas y de la hemorragia digestiva alta no varicosa asociada a cirrosis y fallo hepático agudo (46-48), así como en otras indicaciones menores como el sangrado refractario postesfinterotomía biliar con coagulopatía conocida (49). Existen únicamente dos comunicaciones aisladas del beneficio de su utilización en pacientes con enfermedad de von Willebrand y HDOO por angiodisplasias de intestino delgado o de origen desconocido $(50,51)$.
Dado su marcado carácter protrombótico, se han descrito en la literatura accidentes vasculares a nivel cerebral o coronario asociados a su uso $(51,52)$, especialmente en aquellos pacientes con factores de riesgo cardiovasculares, por lo que en este grupo de pacientes se debe ser cauto a la hora de sentar la indicación.

\section{CONCLUSIÓN}

A la luz de los datos actuales, se puede afirmar que el tratamiento hormonal combinado y otras terapias menos habituales (desmopresina, danazol, antifibrinolíticos, factor VII recombinante) deben ser considerados de mayor utilidad en pacientes con telangiectasia hemorrágica hereditaria, insuficiencia renal crónica terminal y enfermedades hematológicas, fundamentalmente la enfermedad de von Willebrand. En la HDOO por angiodisplasias del intestino delgado o con sospecha de patología vascular sin las enfermedades previamente mencionadas, el octreótido es eficaz y seguro pero está lastrado por su vía de administración parenteral varias veces al día. En un futuro próximo, es probable que el octreótido LAR, la talidomida y su potencial combinación con betabloqueantes no selectivos se perfilen como la piedra angular del tratamiento farmacológico de la HDOO desplazando a la terapia hormonal y al octreótido convencional, por su vía de administración, buena tolerancia y ausencia de efectos secundarios mayores. Son necesarios estudios para confirmar esta hipótesis y determinar la dosis y duración del tratamiento con estos novedosos y prometedores fármacos.

\section{BIBLIOGRAFÍA}

1. Manabe N, Tanaka S, Fukumoto A, Nakao M, Kamino D, Chayama $\mathrm{K}$. Double ballon enteroscopy in patients with GI bleeding of obscure origin. Gastrointest Endosc 2006; 64: 135-40.

2. Lai LH, Wong GL, Chow DK, Lau JY, Sung JJ, Leung WK. Long term follow up of patients with obscure gastrointestinal bleeding after negative capsule endoscopy. Am J Gastroenterol 2006; 101: 1224-9.

3. Hadithi M, Heine GD, Jacobs MA, van Bodegraven AA, Mulder CJ. A prospective study comparing video capsule endoscopy with double-balloon enteroscopy in patients with obscure gastrointestinal bleeding. Am J Gastroenterol 2006; 101: 52-7.

4. Matas JL, Asteinza M, Loscos JM, Fernández S, Ramírez Armengol JA, Díaz Rubio M. Diagnostic yield and safety of capsule endoscopy. Rev Esp Enferm Dig 2006; 98: 666-73.

5. Pérez Cuadrado E, Mas P, Hallal H, Shanabo J, Muñoz E, Ortega I, et al. Double-balloon enteroscopy: A descriptive study of 50 explorations. Rev Esp Enferm Dig 2006; 98: 73-81.

6. Zuckerman GR, Prakash C, Ashkin MP, Lewis BS. AGA technical review: Evaluation and management of occult and obscure gastrointestinal bleeding. Gastroenterology 2000; 118: 20 .

7. Coppola A, Di Stefano V, Tufano A, Nardone G, Amoriello M, Cerbone AM, et al. Long lasting intestinal bleeding in an old patient with multiple vascular abnormalities and Glanzmann's thromboasthenia: 3-year pharmacological management. J Int Med 2002: 252: 271-5.

8. Krikis N, Tziolamos K, Perifanis V, Vakalopoulou S, Karagiannis A, Garipidou, et al. Treatment of recurrent gastrointestinal haemorhage in a patient with von Willebrand's disease with octreotide LAR and propanolol. Gut 2005; 54: 171 . 
9. Harrison DF. Use of estrogen in treatment of familial hemorrhagic telangiectasia. Laryngoscope 1982; 92: 314-20.

10. Panes J, Casadevall M, Fernandez M, Pique JM, Bosch J, Casamitjana $\mathrm{R}$, et al. Gastric microcirculatory changes of portal hypertensive rats can be attenuated by long-term estrogen-progesterone treatment. Hepatology 1994; 20: 1261-70.

11. Nguyen L, Liles DR, Lin PH, Bush RL. Hormone replacement therapy and peripheral vascular disease in women. Vasc Endovascular Surg 2004; 38: 547-56.

12. Van Cutsem E, Rutgeerts P, Vantrappen G. Treatment of bleeding gastrointestinal vascular malformations with oestrogen-progesterone. Lancet 1990; 335: 953-5.

13. Lewis B, Salomon P, Rivera MacMurray S, Kornbluth A, Wenger J, Wayne J. Does hormonal therapy have any benefit for bleeding angiodysplasia? J Clin Gastroenterol 1992; 15: 99-103.

14. Junquera F, Feu F, Papo M, Videla S, Armengol JR, Bordas JM, et al. A multicenter, randomized, clinical trial of hormonal therapy in the prevention of rebleeding from gastrointestinal angiodysplasia. Gastroenterology 2001; 121: 1073-9.

15. Madanick RD, Barkin JS. Hormonal therapy in angiodysplasia: Should we completely abandone its use? Gastroenterology 2002; 123 : 2156.

16. Hodgson H. Hormonal therapy for gastrointestinal angiodysplasia. Lancet 2002; 359: 1630-1.

17. Manzanera MJ, Gutiérrez E, Domínguez Gil B, García JA, González E, Praga M. Hemorragia digestiva por angiodisplasia en pacientes en hemodiálisis. Tratamiento con estrógenos conjugados. Nefrologia 2005; 25: 412-5

18. Tran A, Villeneuve JP, Bilodeau M, Willems B, Marleau D, Fenyves $\mathrm{D}$, et al. Treatment of chronic gastric bleeding from gastric antral vascular ectasia (GAVE) with estrogen-progesterone in cirrhotic patients: An open pilot study. Am J Gastroenterol 1994; 94: 2909-11.

19. Burak KW, Lee SS, Beck PL. Portal hypertensive gastropathy and gastric antral vascular ectasia (GAVE) syndrome. Gut 2001; 49: 86672.

20. Yoshie K, Fujita Y, Moriya A, Kanawa I, Miyamoto Y, Umemura S. Octreotide for severe acute bleeding from portal hypertensive colopathy: A case report. Eur J Gastroenterol Hepatol 2001; 13: 1111-3.

21. Bowers M, McNulty O, Mayne E. Octreotide in the treatment of gastrointestinal bleeding caused by angiodisplasia in two patients with von Willebrand's disease. Br J Hematol 2000; 108: 524-7.

22. Nordquist LT, Wallach PM. Octreotide for obscure gastrointestinal bleeding in an anticoagulated patient. Dig Dis Sci 2002; 47: 1514-5.

23. Blich M, Fruchter O, Edelstein S, Edoute Y. Somatostatin therapy ameliorates chronic and refractory gastrointestinal bleeding caused by diffuse angiodysplasia in a patient on anticoagulation therapy. Scand J Gastroentrerol 2003; 38: 81-3.

24. Nardone G, Rocco A, Balzano T, Budillon G. The efficacy of octreotide therapy in crhonic bleeding due to vascular abnormalities of the gastrointestinal tract. Aliment Pharmacol Ther 1999; 13: 1429-36.

25. Rivera M, Lucero J, Guerrero A, Márquez JL, Montes R, Suner M, et al. Octreotide in the treatment of angiodysplasia in patients with advanced chronic renal failure. Nefrología 2005; 25: 332-5.

26. Kaza Z, Gursel T, Dalgic B, Aslan D. Gastric angiodysplasia in a child with Bernard-Soulier syndrome: Efficacy of octreotide in longterm management. Pediat Hematol Oncol 2005; 22: 223-7.

27. Junquera F, Saperas S, Videla S, Feu F, Vilaseca J, Armengol JR, et al. Long-term efficacy of octreotide in the prevention of recurrent bleeding from gastrointestinal angiodysplasia. Am J Gastroenterol 2007; 102: $254-60$

28. Orsi P, Guatti-Zuliani C, Okolicsanyi L. Long-acting octreotide is effective in controlling rebleeding angiodysplasia of the gastrointestinal tract. Dig Liver Dis 2001; 33: 330-4.

29. Tamagno G, Mioni R, De Carlo E, Maffei P, Rubello D, Sicolo N. Effects of a somatostatin analogue in occult gastrointestinal bleeding: a case report. Dig Liver Dis 2004; 36: 843-6.

30. Molina Infante J, Pérez Gallardo B, González García G, Fernández Bermejo M, Mateos Rodríguez JM, Robledo Andrés P. Octreotide LAR for severe obscure-overt gastrointestinal haemorrhage in highrisk patients on anticoagulation therapy. Gut 2007; 56: 447.

31. Scwartz J, Rozenfeld V, Habot H. Cessation of recurrent bleeding from gastrontestinal angiodysplasia after beta blocker treatment in a patient with a hypertrophic subaortic stenosis: a case history. Angiology 1992; 43: 244-8.

32. Karajeh MA, Hurlstone DP, Stephenson TJ, Ray-Chaudhuri D, Gleeson DC. Refractory bleeding from portal hypertensive gastropathy: A further novel role for thalidomide therapy? Eur J Gastroenterol Hepatol 2006; 18: 545-8.

33. Craanen ME, van Triest B, Verjeihen RH, Mulder CJ. Thalidomide in refractory haemorrhagic radiation induced proctitis. Gut 2006; 55: 1371-2.

34. Hirri HM, Green PJ, Lindsay J. Von Willebrand's disease and angiodysplasia treated with thalidomide. Haemophilia 2006; 12: 285-6.

35. Shurafa M, Kamboj G. Thalidomide for the treatment of bleeding angiodysplasias. Am J Gastroenterol 2003; 98: 221-2.

36. Bauditz J, Schachschal G, Wedel S, Lochs $\mathrm{H}$. Thalidomide for treatment of severe intestinal bleeding. Gut 2004; 53: 609-12.

37. De Konig DB, Drenth JP, Friederich P, Nagengast FM. Thalidomide for the treatment of recurrent gastrointestinal blood loss due to intestinal angiodysplasias. Ned Tijdschr Geneeskd 2006; 150: 1994-7.

38. Kirhkam SA, Lindley KJ, Elawad MA, Blanshard C, Shah N. Treatment of multiple small bowel angiodysplasias causing severe life-threatening bleeding with thalidomide. J Pediatr Gastroenterol Nutr 2006; 42: 585-7.

39. Bauditz J, Lochs H, Voderholzer W. Macroscopic appearance of intestinal angiodysplasias under antiangiogenic treatment with thalidomide. Endoscopy 2006; 38: 1036-9.

40. Hussain I, Saba, Genevieve A. Morelli, Luis A. Logrono. Brief report: Treatment of bleeding in hereditary hemorrhagic telangiectasia vith aminocaproic acid. N Engl J Med 1994; 330: 1789.

41. Philips MD. Stopping bleeding in hereditary telangiectasia. N Engl J Med 1994; 330: 1822-3.

42. Bojan Vujkovac, Janez Lavre, Miso Sabovic. Successful treatment of bleeding from colonic angiodysplasias with tranexamic acid in a hemodialisis patient. Am J Kidney Diseases 1998; 3: 536-8.

43. Haq AU, Glass J, Netchvolodoff CV, Bowen LM. Hereditary hemorrhagic telangiectsia and danazol. Ann Intern Med 1988; 109: 171.

44. Korzenik J, Topazian M, Burdge C, White RI. Danazol in the treatment of GI hemorrhage secondary to hereditary hemorrhagic telangiectasia (abstract). Gastroenterol 1995; 108: A297.

45. Quitt M, Froom P, Veisler A, Falber J, Sova J, Aghai E. The effect of desmopressin on masive gastrointestinal bleeding in hereditary telangiectasia unresponsive to treatment with cryoprecipitate. Arch Intern Med 1990; 150: 1744-6.

46. Romero Castro R, Jiménez Sáenz M, Pellicer Bautista M, Gómez Parra M, Arguelles Arias F, Guerrero Aznar MD, et al. Recombinant-activated factor VII as hemostatic therapy in eight cases of severe hemorrhage from esophageal varices. Clin Gastroenterol Hepatol 2004; 2: 78-84.

47. Bosch J, Tabut D, Bendtsen F, D'Amico G, Albillos A, González Abraldes J, et al. Recombinant factor VIIa for upper gastrointestinal bleeding in patients with cirrhosis: A randomized, double-blind trial. Gastroneterology 2004; 127: 1123-30.

48. Tonkic A, Jukic I, Titlic M, Mise S. Successful treatment of massive acute upper gastrointestinal bleeding in liver failure with activated recombinant factor VII (NovoSeven): A case report. Blood Coagul Fybrinolisis 2005; 16: 385-7.

49. Romero Castro R, Jiménez Sáenz M, Pellicer-Bautista F, Herrerías Gutiérrez JM. Refractory bleeding after endoscopic sphincterotomy: A new indication for recombinant factor VII therapy? Am J Gastroenterol 2004; 99: 2063-5.

50. Meijer K, Peters FT, van der Meer J. Recurrent severe bleeding from gastrointestinal angiodysplasia in a patient with von Willebrand's disease, controlled with recombinant factor VIIa. Blood Coagul Fybrinolisis 2001; 12: 211-3.

51. Basso IN, Keeling D. Myocardial infarction following recombinant activated factor VII in a patient with type 2A von Willebrand disease. Blood Coagul Fybrinolisis 2005; 15: 503-4.

52. Alkyldiz M, Turan I, Ozutemiz O, Batur Y, Ilter T. A cerebrovascular event after single-dose administration of recombinant factor VIIa in a patient with esophageal variceal bleeding. Dig Dis Sci 2006; 51: $1647-9$. 